
\title{
28 Research Suare \\ Conditioned media of mouse macrophages promote neuronal differentiation of mouse hippocampal cells
}

\section{Ayla Batu Öztürk}

Mersin University Faculty of Medicine: Mersin Universitesi Tip Fakultesi

Nail Can Öztürk ( $\square$ nail.ozturk@mersin.edu.tr)

Mersin University https://orcid.org/0000-0001-9459-2120

\section{Furkan Ayaz}

Mersin University Faculty of Science and Letters: Mersin Universitesi Fen Edebiyat Fakultesi

\section{Research Article}

Keywords: Hippocampal cells, Macrophages, Neuromodulation, Neuronal Differentiation, Neurogenesis, Immunotherapy

Posted Date: February 28th, 2022

DOI: https://doi.org/10.21203/rs.3.rs-1383867/v1

License: (c) (i) This work is licensed under a Creative Commons Attribution 4.0 International License. Read Full License 


\section{Abstract}

Understanding how the immune system associates with the pathophysiological hippocampal neurobiology in the neurodegenerative diseases is an emerging topic. Since the cellular population of hippocampal neurogenic niche is extraordinarily diverse, there is still a large gap in our understanding of the interactions between the immune cells and hippocampal neurons under even normal conditions, setting aside the disease conditions. To examine how the hippocampal neurons receive and respond to the cellular factors from the immune cells, we introduced the conditioned cell culture media (CM) of unstimulated, and lipopolysaccharide stimulated RAW 264.7 macrophages to HT22 hippocampal cells during and after they were exposed to neuronal differentiation environment to mimic the states during and after the initiation of the neurogenesis. Then, HT22 cell responses were further examined by assessing immunocytochemical expression of CR protein and mRNA levels of Ascl1, Bdnf, Nrf2 and Rac1 genes via semi quantitative image analysis and q-RT-PCR, respectively. Our results suggest that culturing the HT22 cells with the unstimulated CM of macrophages substantially increased calretinin protein expression, while the latter media containing quite high levels of inflammatory cytokines, measured by ELISA, resulted in decreased protein levels for Calretinin. Additionally, Ascl1 gene expression levels in HT22 cells had similar trends as calretinin protein levels in the presence of CM of stimulated versus unstimulated media. Whereas there was no substantial difference in the gene expression levels for the Bdnf, Nrf2 and Rac1 genes. Overall, our results imply that utilization of HT22 cells and macrophage interplay can be instructive to decipher the molecular mechanisms of cellular communication between the immune system cells and neurons. Our overarching aim is developing immunotherapy strategies by using non-CNS derived immune system cells to modulate the neurogenesis in disease conditions such as Multiple Sclerosis, Alzheimer's and ALS to enable the recovery in the patients.

\section{Introduction}

Since the development and proper functioning of mammalian central nervous system (CNS) depends on the neurogenesis both during and after the development, recent efforts to cope with the CNS related diseases focus on the neuromodulation and neurogenesis studies (Lipp and Bonfanti, 2016; Kempermann, 2019). One of the main hotspots for the neurogenesis research has been centralized on the understanding of the adult hippocampal neurogenesis (AHN). It is one of the two forms of neuronal cell production in the adult mammalian brain, occurring in the sub-granular zone, a layer of dentate gyrus part of hippocampus (Christian et. al., 2020). Although it has been widely accepted that AHN is the main mechanism underlying the normal hippocampal dependent brain functions in rodents, the existence and functional significance of $\mathrm{AHN}$ in human brain is still under debate due to the scarcity of studies stemmed from the ethical concerns and technical difficulties on post-mortem brain tissues. Recent overviews for and against the presence of $\mathrm{AHN}$ in adult human brain can be reviewed elsewhere (Kempermann et. al., 2018; Hagihara et.al., 2019; Petrik and Encinas, 2019; Lucassen et.al., 2020; MorenoJiménez et. al., 2021; Sorrells et. al., 2021). Despite the unsettled discussions over the topic, there are prominent findings featuring the reduction in numbers and maturation of granule neurons in patients with 
various human brain diseases including Alzheimer's Disease and depression which stands as a plausible mechanism behind the memory deficits in neuropathological states in human (Moreno-Jiménez et. al., 2019; Tobin et. al., 2019; Toda et. al., 2019). Such human brain diseases also commonly feature outcomes that overlap with the effects of the neuroinflammation, which points out the importance of the communication between the immune system and nervous system. However, the interactions between the neural and/or neuronal cells in the neurogenic niche of hippocampus and immune cells are poorly understood. In the light of this approach, the motivation of the current study was evaluation of how unstimulated and stimulated inflammatory macrophages' conditioned media, hence the overall soluble factors, affect the hippocampal cells that are undergoing neuronal differentiation.

A long-used approach of mimicking neuroinflammation in rodents via administering either systemic or local lipopolysaccharide (LPS) considerably unveiled the proximity between neuroinflammation and altered neurogenesis (Domínguez-Rivas et. al., 2021). Findings of those in-vivo studies are in consensus with the detrimental effects of LPS-induced microglia on various steps of neurogenic events in the hippocampus (Monje et. al., 2003; Valero et. al., 2014; Tang, 2016; Perez-Dominguez et. al., 2019). So far, the secretion of pro-inflammatory cytokines like TNF- $a$, IL-1 $\beta$ and IL- 6 by acutely activated microglia has been attributed as the main explanation behind inflammation-related hindrance for the adult hippocampal neurogenesis (Domínguez-Rivas et. al., 2021; Tang, 2016; Zonis et. al.,). Further, in-vitro studies by co-culturing adult hippocampal neural stem cells or progenitors with acutely LPS activated microglia, or with LPS-induced conditioned media (CM) demonstrated a substantial reduction in neuronal differentiation (Monje et. al., 2003; Cacci et. al., 2005). In fact, the negative influence of the CNS based immune cells on the neurogenesis was beyond the detrimental effects on the neurogenesis during the disease conditions. Emerging evidence suggest that under normal physiological environment microglia positively regulates hippocampal neurogenesis (Diaz-Aparicio et. al., 2020). But there is still a serious gap in our understanding of the cell-to-cell interactions as well as communications between the immune cells and hippocampal neurons under normal conditions.

Here, we interrogate how neuronal differentiation and homeostasis dynamics are shaped in hippocampal neurons in case they are introduced to unstimulated and inflammatory like cell culture contents of the immune cells. Exposing LPS induced CM of cell line representing the immune system cells such as mouse RAW264.7 macrophages and BV2 microglia on different cell types including neuronal cells has been in use to generate inflammatory models in-vitro (Jonakait et. al., 1996; Monje et. al., 2003; Gugliandolo et. al., 2018; Zong et. al., 2020; Xu et. al., 2021). To address this question, we conducted a set of experiments in which HT22 hippocampal cells were cultivated during and after they were exposed to a neuronal differentiation protocol along with either unstimulated or LPS stimulated CMs of RAW264.7 mouse macrophages. We analyzed the $\mathrm{CM}$ of macrophages by using enzyme-linked immunosorbent assay (ELISA) for the presence of key inflammatory cytokines prior to treating the HT22 cells with them. To evaluate the cellular responses of differentiating and differentiated HT22 cells to those immune cell culture media, we first evaluated the immunocytochemical expression of calretinin (CR), a calciumbinding protein implicated in the regulation of many cellular events in neurons, by cross-group semiquantitative imaging analysis. Additionally, by using quantitative real-time polymerase chain reaction(q- 
RT-PCR), we compared the relative mRNA expression of the genes, that play essential roles in neurogenesis, across the study groups including Brain-derived neurotrophic factor (Bdnf), Achaete-scute homolog 1 (Ascl1), Nuclear factor erythroid 2-related factor 2 (Nrf2) and Ras-related C3 botulinum toxin substrate 1 (Rac1).

\section{Materials And Methods}

\section{Cell culture}

HT-22 Mouse Hippocampal Neuronal and RAW264.7 mouse macrophage cell lines were purchased from Millipore (SCC129) and from American Type Culture Collection (ATCC, Manassas, VA, USA), respectively. We used only the passages between 3 and 8 out of our own HT22 and RAW264.7 cell line stocks for the consistency of the experiments. HT22 cells were maintained in High Glucose Dulbecco's modified Eagle's medium (HG-DMEM; Invitrogen, Carlsbad, CA, USA) supplemented with $10 \%$ (v/v) fetal bovine serum (Atlanta Biologicals, Lawrenceville, GA, USA), $100 \mathrm{U} / \mathrm{mL}$ penicillin, and $100 \mu \mathrm{g} / \mathrm{mL}$ streptomycin at $37^{\circ} \mathrm{C}$ and 5\% (v/v) $\mathrm{CO}_{2}$. RAW264.7 cells were maintained in Roswell Park Memorial Institute media (RPMI 1640 ) supplemented with $10 \%$ fetal bovine serum, $1 \%$ antibiotics $(100 \mu \mathrm{g} / \mathrm{ml}$ penicillin and $100 \mu \mathrm{g} / \mathrm{ml}$ streptomycin) and sodium pyruvate. A mixture of B-27 Plus Neuronal Culture System, a commercial kit, composed of Neurobasal Plus medium and 2\% B-27 Plus Supplement, and N2 supplement (1x, Invitrogen) were used for neuronal differentiation of HT22 cells. To induce the production of inflammatory factors by LPS, RAW264.7 cells were used in $1 \times 10^{6}$ cells/well concentration in $1 \mathrm{ml}$ fresh complete RPMI in 24-well plates. Upon resting overnight in $37 \llbracket \mathrm{C} 5 \% \mathrm{CO}_{2}$ incubator, $1 \mu \mathrm{l} / \mathrm{ml}$ of LPS (1 $\mathrm{mg} / \mathrm{mL}$, Enzo Life Sciences, Salmonella Minnesota R595) was administered in the culture media of RAW264.7cells and cultured for 24 hours (hr). In parallel, a subset of RAW264.7 cells were cultured in the same conditions without the LPS induction, named as unstimulated group. After the experiments with RAW264.7 cells, cell free supernatants of each well were collected and kept at -80凶C prior to the use in ELISA. Triplicates were set up for each experimental condition and these triplicate trials were repeated in

four different independent experiments. A scheme showing the experimental design and groups including treatments and duration is presented (Fig. 1).

\section{Enzyme-linked Immunosorbent Assay}

Culture medium was collected from unstimulated and LPS induced RAW264.7 cells to determine the level of Interleukin 6 (IL-6) and Tumor Necrosis Factor a (TNF-a), Granulocyte-Macrophage Colony-Stimulating Factor (GMCSF) and Interleukin-12 subunit p40 (IL12p40) production by using ELISA. As previously described, each type of cytokine was analyzed according to the manufacturer's instructions (BD Biosciences, CA, USA) (Yüzer and Ayaz, 2019; Ayaz, 2020; Ayaz et. al., 2021).

\section{Immunocytochemistry}


A subset of HT22 cells were seeded in glass bottom imaging dishes (D35-14-1.5-N, Cellvis, Mountain View, CA, USA) at a density of $5 \times 10^{4}$ well to utilize immunophenotypic assessments identical to the experimental design of the study in the schema (Fig. 1). Following differentiation and treatments, HT22 cells were first washed three times with PBS and fixed in 4\% paraformaldehyde solution (4\% paraformaldehyde, $\mathrm{pH} 7.4$, in PBS) for 10 min at room temperature. The cells were then washed three times with PBS, permeabilized in $0.25 \%$ Triton X-100 (Sigma-Aldrich) in PBS for 15 min and washed again three times with PBS. Next, the cells were blocked for $1 \mathrm{~h}$ at room temperature in a staining buffer (used as a buffer for primary and secondary antibody staining as well) including $5 \%$ normal goat serum (\#005-000-121, ImmunoResearch, West Grove, PA), 1\% bovine serum albumin (112018, Sigma-Aldrich) and $0.025 \%$ Triton X-100 to reduce nonspecific binding. The cells were then incubated with different primary antibodies/antiserum (1:500, rabbit anti Calretinin anti serum, 7697, Swant, Switzerland) in the staining buffer at $4^{\circ} \mathrm{C}$ overnight. Following sufficient washing, the cells were incubated with the secondary antibody against the primary antibody species in the staining buffer (1:750, Alexa Fluor 488 AffiniPure Goat Anti-Rabbit IgG $(\mathrm{H}+\mathrm{L})$, ImmunoResearch) for $1.5 \mathrm{~h}$ at $37^{\circ} \mathrm{C}$ in dark at $\mathrm{RT}$, washed in cold PBS for several times and counterstained with 4',6-diamidino-2-phenylindole (DAPI- A-T-specific DNA stain at $350 \mathrm{~nm}$ wavelength, Invitrogen). The morphologies were captured under fluorescent microscope (Nikon Ti-Eclipse, Tokyo, Japan) interfaced with a Digital-Sight camera via NIS-Elements Software from Nikon.

\section{Quantitative Image Analysis}

Preliminary observations in the groups showed a differential fluorescent intensity among the CR immune reactive cells. Therefore, instead of performing the analysis on an immune-positive or negative cell counting fashion, we utilized from a scoring in which the intensity of specific fluorescent dye in each cell representing the immunocytochemical expression of CR protein (Singh et.al., 2009; Chen et.al., 2013). The presence of positive staining was judged based on the camera's capture of a distinguishable image above background (black) level and by overlay of DAPI staining. Fluorescent images at 4 adjacent areas per each quadrant of the imaging dishes ( $n=4$ per group) were captured at 40X objective magnification with the consistent setup and exposure time according to the base determined in the control group (G1). Image capturing of the adjacent areas at the quadrants were performed in a clockwise manner to avoid bias. A schema of the imaging routine is shown (Supplementary Fig). Alexa Fluor 488 conjugated dyestained fluorescent images were converted to 8-bit color format and analyzed with Image $\mathrm{J}$ (National Institutes of Health, Bethesda, MD). Each cell with clear boundary per picture was outlined using the freehand circle tool of ImageJ. Then the fluorescent intensity of CR immunostaining of each outlined cell was measured.

The intensity values of all the cells (20-100 cell/captured image) per captured image $(n=4$ image/group) were ranked into three equal intervals of which represented as Weak - 1 -; Moderate - 2 -; and High -3 . Overall, immunocytochemical score of each dish was obtained by the following formula: 3 $\times$ percentage of highly stained cells $+2 \times$ percentage of moderately stained cells + percentage of weakly stained cells, giving a range of $0-250$. 


\section{Rna Extraction, Cdna Synthesis And Q-rt-pcr}

Total RNA from the experimental group of cells (G1-G6, shown in Fig. 1) were extracted by using RNeasy Mini isolation kit (74106, Qiagen, Hilden, Germany), according to the manufacturer's instructions. Prior to the cDNA synthesis, $1.5 \mu \mathrm{l}$ of each extracted RNA sample was used to measure the concentration and purity of RNA by using NanoDrop One spectrophotometer (NanoDrop Technologies Inc., Wilmington, DE by Thermo Scientific). The sample absorbance was detected at $230 \mathrm{~nm}, 260 \mathrm{~nm}$ and $280 \mathrm{~nm}$. 260/280 ratio was used to predict RNA purity along with the nucleic acid concentrations in $n g / \mu \mathrm{L}$, while $260 / 230$ ratio was utilized to check the presence of possible contaminants absorbing at $230 \mathrm{~nm}$ or less. RNA samples, only with above 2.0 values of $260 / 280$ and $260 / 230$ ratios were used for cDNA synthesis. Immediately after the quantity and purity check, RNA samples were diluted to $250 \mathrm{ng} / \mu \mathrm{l}$ final concentration, aliquoted and stored in -80冈C. All the RNA samples were reverse transcribed at the same time with the High-Capacity Reverse Transcription kit (4368814, Applied Biosystems), using $500 \mathrm{ng}$ of total RNA per $1 \mu$ lMultiScribe ${ }^{\text {TM }}$ Reverse Transcriptase enzyme in the kit and stored in aliquots in $-80 \rrbracket$ C.Primer pairs were designed by using Snap Gene Viewer software (Table 1). Specificity and quality of designed primers were checked by using Primer-BLAST and IDT SciTools Web (Integrated DNA Technologies, Coralville, IA, USA). Primers were synthesized by Integrated DNA Technologies (Integrated DNA Technologies, Coralville, IA, USA). Q-RT-PCR was performed in $15 \mu$ reaction volume with $2 x$ SsoFast Eva Green Supermix (1725201, Bio-Rad), at $95^{\circ} \mathrm{C}$ for $5 \mathrm{~min}$, followed by 40 cycles of $95^{\circ} \mathrm{C}$ for $5 \mathrm{~s}$ and $51^{\circ} \mathrm{C}$ for $20 \mathrm{~s}$, followed by real-time melt analysis to verify product specificity in technical triplicates using LighCycler480 II instrument (Roche). Threshold cycle values were determined using the comparative CT $\left(2^{-\triangle \triangle C T}\right)$ method (Livak and Schmittgen, 2001). normalized to mouse hypoxanthine-guanine phosphoribosyl transferase (Hprt).

Table 1

Primers for q-RT-PCR

\begin{tabular}{|lll|}
\hline Gene Symbol & Forward (5囚-3】) & Reverse (3】 -5】) \\
\hline Hprt & CAGCGTCGTGATTAGCGATGAT & GAGCAAGTCTTTCAGTCCTGTCCA \\
Bdnf & AGCTGCCTTGATGTTTACTTTGAC & GTCCGTGGACGTTTACTTCTTTCA \\
Ascl1 & ACTTGAACTCTATGGCGGGTT & CCAGTTGGTAAAGTCCAGCAG \\
Nrf2 & AAGAATAAAGTCGCCGCCCA & AGATACAAGGTGCTGAGCCG \\
Rac1 & GGTAGGTGATGGGAGTCAGC & CTGAAGTGCGACACCACTGT \\
\hline
\end{tabular}

\section{Statistical analysis}

Statistical analysis was performed using the free trial version of IBM SPSS Statistics 26 software. ONEWay ANOVA test was used to address whether differences exist among the mean immunocytochemical score and target mRNA levels of the groups. Alternations in paired groups were determined by using 
Bonferroni post-hoc test. Data were presented as mean \pm SD differences. $p<0.05$ was considered statistically significant.

\section{Results}

\section{Cytokine levels in the CM of unstimulated and LPS- stimulated RAW264.7 cells}

Prior to culturing HT22 cells with unstimulated or LPS-stimulated CMs of RAW264.7 mammalian macrophages, production levels of TNF, IL6, GMCSF and IL12p40, inflammatory cytokines were measured in the supernatants of the media by ELISA. While there were no detectable levels of these cytokines in the unstimulated media of RAW264.7 cells, there were distinctly high levels of each measured cytokine in the LPS stimulated cells' CM (Fig. 2).

\section{Comparison Of Immunocytochemical Cr Expression Among The Groups}

Semi-quantitative analysis of immunocytochemical expression of CR protein between multiple groups was conducted based on the fluorescence intensity of specific immunoreactivity in the cells. Fluorescent intensity or immunocytochemical scores of CR expression in HT22 cell groups that were exposed to unstimulated CM of RAW264.7 macrophages, during (G3) and after (G5) subjected to the differentiation protocol were significantly higher than the following HT22 cell groups that were exposed to; CM of LPS stimulated RAW264.7 cells during ( $G 3$ vs $G 4$ and $G 6 ; p<0.001$ ) and after they were subjected to the differentiation protocol ( $G 5$ vs $G 4$ and $G 6, p<0.001$ ), differentiation protocol only (G3 vs $G 2, p<0.001 ; G 5$ vs $G 2, p<0.001$ ) and control media HG-DMEM (G3 vs $G 1, p<0.01 ; G 5$ vs $G 1, p<0.01$ ) (Fig. 3). There was no significant difference $(p>0.05)$ between the following paired groups; $G 1$ vs $G 2$, G2 vs G4, G4 vs G6 (Fig. 3).

\section{Comparison of mRNA expression of neurogenesis related target genes among the groups}

Quantitative real-time PCR analysis of Bdnf, Ascl1, Rac1 and Nrf2 mRNA levels in terms of fold changes $\left(2^{-\triangle \triangle C T}\right)$ across the experimental groups (G1-G6) are shown in the bar graphs, respectively (Fig. 4A-D). Expression of the neurogenesis related or neural target genes in each group were normalized to the expression of Hprt reference gene for comparative analysis. Error bars represent the standard deviation of fold alternations of four biological replicates within the groups. Significance and F values of ONE-Way ANOVA test are shown at the right upper corner of each section (Fig. 4A-D) which indicate whether differences exist among the mean mRNA fold changes of different groups. Horizontal lines between the bars indicate statistically significant $(p<0.05)$ pair-wise alternations, analyzed via Bonferroni post-hoc test. Pair-wise relations with significance level of $p>0.05$ were not pointed out, except three that were close the significance level of $p=0.05$, were highlighted in red (Fig. 4A). 
Bdnf mRNA levels relative to the control group were not significantly altered in the HT22 cells that were subjected to either differentiation media ( $G 2$ vs $G 1, p>0.05$ ) or a mix of differentiation and CM of unstimulated macrophages ( $G 3$ vs $G 1, p>0.05$ ) for 24 hr., while a notable increment was evident when cultured with $\mathrm{CM}$ of unstimulated macrophages ( $\mathrm{G} 5 \mathrm{vs} \mathrm{G} 1, \mathrm{p}=0.052$ ) for $24 \mathrm{hr}$. following the differentiation protocol (Fig. 4A). Culturing HT22 cells with CM of LPS-induced macrophages along with the differentiation media distinctly reduced Bdnf mRNA expression compared to the control group (G4 vs $G 1, p<0.01$ ), and to the groups cultured with the differentiation media only (G4 vs $G 2, p<0.001$ ) and CM of macrophages mixed with the differentiation media (G4 vs $G 3, p<0.001$ ). A notable but statistically non-significant reduction following the differentiation protocol, in the group that cultured with CM of LPS induced macrophages as compared to the group cultured with CM of unstimulated macrophages (G6 vs $G 5, p=0.082$ ) was found. There was no difference between the control group and the group that was cultured with the $\mathrm{CM}$ of unstimulated macrophages after the differentiation protocol (G6 vs $G 1, p>0.05$ ) (Fig. 4A).

A substantial increment trend in theAscl1 mRNA fold change was apparent, in cells that were incubated with either differentiation media ( $G 2$ vs $G 1, p<0.01$ ) or $C M$ of unstimulated macrophages both during (G3 vs $G 1, p<0.001$ ) and following the differentiation protocol ( $G 5$ vs $G 1, p<0.001$ ) compared to the control group (Fig. 4B). There was also a distinct Ascl1 fold increment in HT22 cells that were subjected to differentiation media mixed with $\mathrm{CM}$ of unstimulated macrophages compared to the differentiation media only (G3 vs G2, p < 0.01) (Fig. 4B). While it was not different than the control group (G4 vs G1, p> 0.05 and $G 6$ vs $G 1, p>0.05$ ), cells that were treated with CM of LPS-induced macrophages had significantly lower levels of Ascl1 mRNA fold change, compared to differentiating (G4 vs G2, $p<0.05$ and G4 vs G3, $p<0.001$ ) and differentiated (G6 vs G5, $p<0.001$ ) groups (Fig. 4B). Fold change levels for Rac1 $(p>0.05$, Fig. 4C) and Nrf2 ( $p>0.05$, Fig. 4D) target genes did not show any significant alternation among the groups, when tested by ONE-Way ANOVA analysis.

\section{Discussion}

In our current study, we are presenting quantitative cellular and molecular evaluations of how some neural regulators act in mouse HT22 hippocampal neurons in response to both the unstimulated and LPS induced conditioned cell culture environment of mouse RAW 264.7 macrophages, which were not previously explored in tandem. We utilized HT22 cell line to conduct the studies because it has been a consistent and well-established cell line representing hippocampus tissue and has been frequently used in modeling pathological features associated with the neurodegenerative diseases (Liu et. al., 2009; Wang et. al., 2019; Park et. al., 2019). We utilized from the CMs of a non-CNS originated type of immune cell to culture with the hippocampal cells in our study design. Culturing neural or neuronal cells with proinflammatory cytokines rich CM of LPS-induced immune cells, especially with BV-2 microglial cell line appears as a useful strategy of mediating neuroinflammatory environment (Jonakait et. al., 1996; Monje et. al., 2003; Zong et. al., 2020; Fernández-Calle et. al., 2020; Xu et. al., 2021). As microglial cells are the principle immune guards in the neurogenic niches of adult brain, they are regarded as the main actors in many in-vivo studies evaluating neuron to immune cell communications. Meanwhile, immune modulation 
capacity of another type of immune cell, macrophages is also similar with microglia. Depending on the stimulus, both immune cells protect their surrounding microenvironment and eliminate the pathogens and other threats with a battery of pro- and anti-inflammatory cytokines and factors. For example, as evident by our ELISA analysis, we measured high levels of canonical inflammatory cytokines in the CM of LPS induced RAW 264.7 cells, while the unstimulated media was devoid of those elements. Similar immune responses regarding most of those cytokines were also found in the CM of LPS induced BV-2 cells by the previous studies (Li et. al.,2016; Ji et. al., 2019). We utilized from a macrophage type that had peripheral origin since during the clinical applications of neuromodulatory immunotherapy the source of the immune system cells and/or their conditioned media will be the peripheral immune system instead of invasive approaches to get microglia from the CNS.

A point worthy of mentioning here is that though located far from the CNS, dialogue between the macrophages and the neurons in the gastrointestinal tract is crucial for maintaining neuronal health both during homeostasis and pathology (Gabanyi et. al., 2016; Meroni et. al., 2019; Viola and Boeckxstaens, 2020;). Based on their immunomodulatory potentials, both macrophages and microglia can be valuable assets for immunotherapeutic approaches to the neurodegenerative diseases. Although microglia are the main immune cells in CNS, yet their use in such an approach in human, has a major drawback because obtaining microglia is not practical or plausible. In this context, using monocytes/macrophages from the periphery in humans, which is easier and less invasive to obtain compared to obtaining the microglial cells, may be a more advantageous and promising strategy. From this point of view, we preferred using macrophages over microglial cells to interrogate the neural reactions of HT22 cells to the contents of the immune cell culture.

In the light of this aim, first, we found that culturing HT22 cells with the CM of unstimulated RAW 264.7 macrophages increased the CR expression in HT22 cells, while culturing them with the CM of LPS induced macrophages generated the opposite effect. It is important whether this outcome (increased vs decreased) of CR expression in HT22 neurons via CMs of unstimulated and LPS induced macrophages is meaningful. As $\mathrm{CR}$ is a $\mathrm{Ca}^{2+}$ modulator, the balance of its expression in neurons is crucial for intracellular $\mathrm{Ca}^{2+}$ homeostasis (Rogers, 1987; Qi et. al., 2021). Reduction in CR expression by HT22 neurons due to inflammatory factors from the CM of the LPS induced macrophages can be interpreted as an indicator of aberrant intracellular $\mathrm{Ca}^{2+}$ balance. Besides, having the opposite trend of $\mathrm{CR}$ expression in the groups of HT22 cells exposed to CMs of unstimulated vs LPS induced macrophages appears as a consistent outcome. Nevertheless, there are reports in which the expression of CR was preserved, increased, or decreased depending on the brain region in different disease models (Yang et. al., 2018; Zallo et. al., 2018; Petrache et. al., 2019). Additionally, detection of CR protein is frequently used to mark the early phase immature neurons to help measuring the limits of adult neurogenesis in the brains of different species including rodents, primates and human (Brandt et. al., 2003; Moreno-Jiménez et. al., 2019; MorenoJiménez et. al., 2021). With regards to the role of $\mathrm{CR}$ attributed as a cell marker of early immature neurons in AHN or early post-mitotic phase of neuronal differentiation, our findings support that cultivating HT22 
cells with CM of unstimulated macrophages provoked the neuronal differentiation, whereas the CM of LPS stimulated inflammatory macrophages hindered the progress of differentiation.

A stark increase of the Ascl1 mRNA levels in HT22 cells which were cultured with CM of unstimulated RAW 264.7 macrophages is concordant with the findings of cellular CR expression. Existing body of literature fairly represents the positive influence of Ascl1 expression on the regulation of neural differentiation or neurogenesis (Yoo et. al., 2011; Karow et. al., 2012; Chouchane et. al., 2017; Vasan et. al., 2021). In this context, substantial elevation of Ascl1 mRNA expression in HT22 cells upon exposure to the $\mathrm{CM}$ of unstimulated macrophages strongly indicates a positive progression in the progress of neurogenesis, which is also supported by $\mathrm{CR}$ expression trends in our results. There was also a notable incremental trend in the Bdnf mRNA levels in HT22 cells that were exposed to the $\mathrm{CM}$ of unstimulated macrophages, however this was barely significant in our statistical analysis. In parallel, reduction of Bdnf mRNA levels in HT22 cells that were cultured with the CM of inflammatory macrophages suggests a decrease in neural activity and perhaps indication of neuroinflammation or disrupted cellular homeostasis. There is a plenty of literature reporting reduced levels of Bdnf in the blood and/or brain of patients with psychiatric and neurodegenerative disorders, and in the experimental studies of neuroinflammation and our results overlap with those reports (Lima Giacobbo et. al., 2019).

Next, we intended to explore the cellular homeostasis further, in HT22 cells that were exposed to the CMs of RAW 264.7 macrophages. On this basis, we interrogated the status of cellular homeostasis by means of Nrf2 mRNA levels which is critical for facilitating the cellular defense to endogenous and exogenous cellular stressors including oxidants and inflammatory factors according to the recent reports (Itoh et. al., 2010; Bryan et. al., 2013; Suzuki and Yamamoto, 2017). Besides, recent studies attributed novel roles to Nrf2 expression in the regulation of neural stem/progenitor cell functioning with aging and the balance of a-synuclein through proteostasis (Anandhan et. al., 2021; Baxter et. al., 2021). However, we found that there was no significant difference in Nrf2 transcription in HT22 cells between of the experimental groups. Why neither of the CMs of macrophages in our experimental setting, markedly affected the transcription levels of this key cellular homeostasis regulator, while accumulating meaningful responses by means of other examined factors is an important question that requires further investigation.

If we were to investigate these issues in an in-vivo setting, then, we could have obtained quite different levels of the same elements of interests. This is highly likely because neurogenic niche in sub-granular zone of hippocampus can be considered as a granule neuron factory which harbors a wide spectrum of cell types in addition to the neuronal cells undergoing neurogenesis, including but not limited to inhibitory neurons and mossy cells, glial cells, astrocytes, oligodendrocytes, and microglia (Ekdahl, 2012; Song et. al., 2016; Falk and Götz, 2017; Yeh et. al., 2018). Since all these cells in the neurogenic niche exert their effect on neuronal differentiation by releasing different molecules including neurotransmitters, growth factors, morphogens, cytokines, hormones etc., differentiating hippocampal neurons are exposed to dozens of other cellular factors at a time. Therefore, abilities of in-vivo studies or coculturing in-vitro studies fall short to isolate the single cell type-based responses of differentiating hippocampal cells to a certain stimulus in the mid of crowded multicellular dialogues. By exposing the CMs of one cell type to 
another, rather than coculturing, we were able to determine some means of cellular communication between the neuronal cells and macrophages. These can be considered as cellular monologs rather than dialogues.

Therefore, we can extrapolate that cellular homeostasis through Nrf2 modulation may not be potent in differentiating hippocampal cells without the concurrent cellular factors that occur in the natural neurogenic hippocampal niche. One can facilitate a variety of smart in-vitro designs to interrogate the Nrf2 dependent dialogue between hippocampal neurons and immune cells. In this study, we had the chance to assess the mRNA expression level of Rac1, another key neural/neuronal regulator. But we found no significant difference of Rac1 expression in comparison to the other groups. We interpret the results of Rac1 expression similar to those of Nrf2. Further analyses are needed with different cellular factors and cell groups to decipher possible role of other factors from different cell types that might modulate Rac1 and Nrf2 expression levels that play crucial role in neurogenesis.

\section{Conclusion}

While the breadth of possible neuron-immune cell interactions that can be studied is vast, in this study we were able to isolate only a few in vitro cellular monologs, limited to the responses of HT22 neurons to the conditioned cell culture media of RAW 264.7 macrophages. Our findings decipher that the signaling molecules in the conditioned media from the unstimulated macrophages lead to neuronal differentiation in HT22 cells. Further efforts are anticipated to address deeper questions of how macrophages interact with the hippocampal neurons. Further studies are required to determine the soluble factors and signaling systems that function through cell-to-cell interaction that enable neuromodulation by different immune system cells and supporting cells in the CNS. Isolation of the immune system cells and utilization of them in the immunotherapy may open new venues for the field of neuromodulation. Our group presents the preliminary results supporting the positive roles of macrophage based conditioned medium that can be utilized against neurodegenerative diseases.

\section{Abbreviations}

AHN, adult hippocampal neurogenesis; Ascl1, Achaete-scute homolog 1; Bdnf, Brain-derived neurotrophic factor; cDNA, complementary DNA; CNS, central nervous system; CM, conditioned media; GMCSF, Granulocyte-Macrophage Colony-Stimulating Factor; ELISA, enzyme-linked immunosorbent assay; HGDMEM, High glucose Dulbecco's modified Eagle's media; IL-6, Interleukin 6; IL12p40, Interleukin-12 subunit p40; LPS, lipopolysaccharide; Nrf2, nuclear factor erythroid 2-related factor 2; Rac1, Ras-related C3 botulinum toxin substrate 1; TNF-a, Tumor Necrosis Factor $\mathbf{a}$; q-RT-PCR, quantitative real-time polymerase chain reaction

\section{Declarations}




\section{Data Availability}

The datasets generated during and analyzed during the current study are available from the corresponding author on reasonable request.

Ethics approval and consent to participate (Human Ethics, Animal Ethics or Plant Ethics)

Not Applicable

\section{Consent for publication}

All authors approved the final version of the manuscript.

\section{Availability of data and materials}

Not Applicable

\section{Competing Interest}

The authors declare that they have no competing interest.

\section{Funding}

This work was supported by Scientific Research Projects Unit of Mersin University 2018-1-AP4-2875 and 2020-1-AP5-4104 to NCÖ. ABÖ was supported by 100/2000 scholarship in the Neurogenetics field funded by Council of Higher Education in Turkey

\section{Author' contributions}

All authors contributed to the study conception and design. Ayla Batu Öztürk (PhD Candidate)- data collection and analysis, figure sketching and manuscript writing, Nail Can Öztürk (PhD, Assistant Professor) -project development, data analysis, supervision, manuscript writing. Furkan Ayaz (PhD, Associate Professor) - data analysis, supervision, manuscript writing.

We greatly appreciate the guidance and support of Professor Feng C. Zhou, Emeritus of Anatomy and Cell Biology \& Medical Neurobiology, Indiana Univ. Sch. Med and Prof. Dr. Juan Anguita from CICBiogune.

\section{References}

1. Anandhan A, Kirwan KR, Corenblum MJ, Madhavan L (2021) Enhanced NRF2 expression mitigates the decline in neural stem cell function during aging. Aging Cell 20:e13385. doi: 10.1111/acel.13385

2. Ayaz F (2020) Ruthenium Bipyridyl Dithiocyanate Complex Exerted Adjuvant Activity on the Activated Mammalian Macrophages in vitro. Inflammation 43:1120-1126. doi: 10.1007/s10753-020-01199-9 
3. Ayaz F, Demir D, Bölgen N (2021) Injectable chitosan cryogel microspheres with biocompatible properties on mammalian macrophages in vitro. J Mater Sci 56:17268-17277. doi: 10.1007/s10853021-06399-8

4. Baxter PS, Márkus NM, Dando O, He X, Al-Mubarak BR, Qiu J (2021) Hardingham GE. Targeted derepression of neuronal Nrf2 inhibits a-synuclein accumulation. Cell Death Dis 12:218. doi:10.1038/s41419-021-03507-z

5. Brandt MD, Jessberger S, Steiner B, Kronenberg G, Reuter K, Bick-Sander A, von der Behrens W, Kempermann G (2003) Transient calretinin expression defines early postmitotic step of neuronal differentiation in adult hippocampal neurogenesis of mice. Mol Cell Neurosci 24:603-613. doi: 10.1016/s1044-7431(03)00207-0

6. Bryan HK, Olayanju A, Goldring CE, Park BK (2013) The Nrf2 cell defence pathway: Keap1-dependent and -independent mechanisms of regulation. Biochem Pharmacol 85:705-717. doi:

10.1016/j.bcp.2012.11.016

7. Cacci E, Claasen JH, Kokaia Z (2005) Microglia-derived tumor necrosis factor-alpha exaggerates death of newborn hippocampal progenitor cells in vitro. J Neurosci Res 80:789-797. doi: 10.1002/jnr.20531

8. Chen Y, Ozturk NC, Zhou FC (2013) DNA methylation program in developing hippocampus and its alteration by alcohol. PLoS ONE 8:e60503. doi: 10.1371/journal.pone.0060503

9. Chouchane M, Melo de Farias AR, Moura DMS, Hilscher MM, Schroeder T, Leão RN, Costa MR (2017) Lineage Reprogramming of Astroglial Cells from Different Origins into Distinct Neuronal Subtypes. Stem Cell Reports 9:162-176. doi:10.1016/j.stemcr.2017.05.009

10. Christian KM, Ming GL, Song H (2020) Adult neurogenesis and the dentate gyrus: Predicting function from form. Behav Brain Res 379:112346. doi: 10.1016/j.bbr.2019.112346

11. Diaz-Aparicio I, Paris I, Sierra-Torre V, Plaza-Zabala A, Rodríguez-Iglesias N, Márquez-Ropero M, Beccari S, Huguet P, Abiega O, Alberdi E, Matute C, Bernales I, Schulz A, Otrokocsi L, Sperlagh B, Happonen KE, Lemke G, Maletic-Savatic M, Valero J, Sierra A (2020) Microglia Actively Remodel Adult Hippocampal Neurogenesis through the Phagocytosis Secretome. J Neurosci 40:1453-1482. doi: 10.1523/JNEUROSCI.0993-19.2019

12. Domínguez-Rivas E, Ávila-Muñoz E, Schwarzacher SW, Zepeda A (2021) Adult hippocampal neurogenesis in the context of lipopolysaccharide-induced neuroinflammation: A molecular, cellular and behavioral review. Brain Behav Immun 97:286-302. doi: 10.1016/j.bbi.2021.06.014

13. Ekdahl CT (2012) Microglial activation - tuning and pruning adult neurogenesis. Front Pharmacol 9:3-41. doi:10.3389/fphar.2012.00041

14. Falk S, Götz M (2017) Glial control of neurogenesis. Curr Opin Neurobiol 47:188-195. doi: $10.1111 / \mathrm{nmo} .13843$

15. Fernández-Calle R, Galán-Llario M, Gramage E, Zapatería B, Vicente-Rodríguez M, Zapico JM, de Pascual-Teresa B, Ramos A, Ramos-Álvarez MP, Uribarri M, Ferrer-Alcón M, Herradón G (2020) Role of 
RPTP $\beta / \zeta$ in neuroinflammation and microglia-neuron communication. Sci Rep 10:20259. doi: 10.1038/s41598-020-76415-5

16. Gabanyi I, Muller PA, Feighery L, Oliveira TY, Costa-Pinto FA, Mucida D (2016) Neuro-immune Interactions Drive Tissue Programming in Intestinal Macrophages. Cell 164:378-391. doi: 10.1016/j.cell.2015.12.023

17. Gugliandolo A, Pollastro F, Grassi G, Bramanti P, Mazzon E (2018) In Vitro Model of Neuroinflammation: Efficacy of Cannabigerol, a Non-Psychoactive Cannabinoid. Int J Mol Sci 19: 1992. doi: $10.3390 /$ ijms 19071992

18. Hagihara H, Murano T, Ohira K, Miwa M, Nakamura K, Miyakawa T (2019) Expression of progenitor cell/immature neuron markers does not present definitive evidence for adult neurogenesis. Mol Brain 12:108. doi: 10.1186/s13041-019-0522-8

19. Itoh K, Mimura J, Yamamoto M (2010) Discovery of the negative regulator of Nrf2, Keap1: a historical overview. Antioxid Redox Signal 13:1665-1678. doi: 10.1089/ars.2010.3222

20. Ji Y, Wang X, Kalicki C, Menta BW, Baumgardner M, Koppel SJ, Weidling IW, Perez-Ortiz J, Wilkins HM, Swerdlow RH (2019) Effects of Microglial Cytokines on Alzheimer's Disease-Related Phenomena. J Alzheimers Dis 67:1021-1034. doi:10.3233/JAD-180820

21. Jonakait GM, Luskin MB, Wei R, Tian XF, Ni L (1996) Conditioned medium from activated microglia promotes cholinergic differentiation in the basal forebrain in vitro. Dev Biol 177:85-95. doi: 10.1006/dbio.1996.0147

22. Karow M, Sánchez R, Schichor C, Masserdotti G, Ortega F, Heinrich C, Gascón S, Khan MA, Lie DC, Dellavalle A, Cossu G, Goldbrunner R, Götz M, Berninger B (2012) Reprogramming of pericyte-derived cells of the adult human brain into induced neuronal cells. Cell Stem Cell 11:471-476. doi: 10.1016/j.stem.2012.07.007

23. Kempermann G, Gage FH, Aigner L, Song H, Curtis MA, Thuret S, Kuhn HG, Jessberger S, Frankland PW, Cameron HA, Gould E, Hen R, Abrous DN, Toni N, Schinder AF, Zhao X, Lucassen PJ, Frisén J (2018) Human Adult Neurogenesis: Evidence and Remaining Questions. Cell Stem Cell 23:25-30. doi: 10.1016/j.stem.2018.04.004

24. Kempermann G (2019) Environmental enrichment, new neurons and the neurobiology of individuality. Nat Rev Neurosci 20:235-245. doi: 10.1038/s41583-019-0120-x

25. Li N, Liu BW, Ren WZ, Liu JX, Li SN, Fu SP, Zeng YL, Xu SY, Yan X, Gao YJ, Liu DF, Wang W (2016) GLP-2 Attenuates LPS-Induced Inflammation in BV-2 Cells by Inhibiting ERK1/2, JNK1/2 and NF-KB Signaling Pathways. Int J Mol Sci 17:190. doi: 10.3390/ijms17020190

26. Lima-Giacobbo B, Doorduin J, Klein HC, Dierckx RAJO, Bromberg E, de Vries EFJ (2019) Brain-Derived Neurotrophic Factor in Brain Disorders: Focus on Neuroinflammation. Mol Neurobiol 56:3295-3312. doi: 10.1007/s12035-018-1283-6

27. Lipp HP, Bonfanti L (2016) Adult Neurogenesis in Mammals: Variations and Confusions. Brain Behav Evol 87:205-221. doi: 10.1159/000446905 
28. Liu J, Li L, Suo WZ (2009) HT22 hippocampal neuronal cell line possesses functional cholinergic properties. Life Sci 84:267-271. 10.1016/j.Ifs.2008.12.008

29. Livak KJ, Schmittgen TD (2001) Analysis of relative gene expression data using real-time quantitative PCR and the 2(-Delta Delta C(T)) Method. Methods 25:402-408. doi: $10.1006 /$ meth.2001.1262

30. Lucassen PJ, Fitzsimons CP, Salta E, Maletic-Savatic M (2020) Adult neurogenesis, human after all (again): Classic, optimized, and future approaches. Behav Brain Res 381:112458. doi: 10.1016/j.bbr.2019.112458

31. Meroni E, Stakenborg N, Viola MF, Boeckxstaens GE (2019) Intestinal macrophages and their interaction with the enteric nervous system in health and inflammatory bowel disease. Acta Physiol (Oxf) 225:e13163. doi:10.1111/apha.13163

32. Monje ML, Toda H, Palmer TD (2003) Inflammatory blockade restores adult hippocampal neurogenesis. Science 302:1760-1765. doi: 10.1126/science.1088417

33. Moreno-Jiménez EP, Flor-García M, Terreros-Roncal J, Rábano A, Cafini F, Pallas-Bazarra N, Ávila J, Llorens-Martín M (2019) Adult hippocampal neurogenesis is abundant in neurologically healthy subjects and drops sharply in patients with Alzheimer's disease. Nat Med 25:554-560. doi: 10.1038/s41591-019-0375-9

34. Moreno-Jiménez EP, Terreros-Roncal J, Flor-García M, Rábano A, Llorens-Martín M (2021) Evidences for Adult Hippocampal Neurogenesis in Humans. J Neurosci 41:2541-2553. doi: 10.1523/JNEUROSCI.0675-20.2020

35. Park J, Park J, Kim K (2019) Neuroprotective effects of myristargenol A against glutamate-induced apoptotic HT22 cell death. RSC Adv 9:31247-31254. doi: 10.1039/C9RA05408A

36. Perez-Dominguez M, Ávila-Muñoz E, Domínguez-Rivas E, Zepeda A (2019) The detrimental effects of lipopolysaccharide-induced neuroinflammation on adult hippocampal neurogenesis depend on the duration of the pro-inflammatory response. Neural Regen Res 14:817-825. doi: 10.4103/16735374.249229

37. Petrache AL, Rajulawalla A, Shi A, Wetzel A, Saito T, Saido TC, Harvey K, Ali AB (2019) Aberrant Excitatory-Inhibitory Synaptic Mechanisms in Entorhinal Cortex Microcircuits During the Pathogenesis of Alzheimer's Disease. Cereb Cortex 29:1834-1850. doi:10.1093/cercor/bhz016

38. Petrik D, Encinas JM (2019) Perspective: Of Mice and Men - How Widespread Is Adult Neurogenesis? Front Neurosci 13:923. doi:10.3389/fnins.2019.00923

39. Qi Y, Cheng H, Wang Y, Chen Z (2021) Revealing the Precise Role of Calretinin Neurons in Epilepsy: We Are on the Way. Neurosci Bull 10: 1007/s12264-021-00753-1. Doi: 10.1007/s12264-021-00753-1

40. Rogers JH (1987) Calretinin: a gene for a novel calcium-binding protein expressed principally in neurons. J Cell Biol 105: 1343-53. Erratum in: J Cell Biol 1990 110: 1845. doi: 10.1083/jcb.105.3.1343

41. Singh RP, Shiue K, Schomberg D, Zhou FC (2009) Cellular epigenetic modifications of neural stem cell differentiation. Cell Transpl 18:1197-1211. doi: 10.3727/096368909X12483162197204 
42. Song J, Olsen RH, Sun J, Ming GL, Song H (2016) Neuronal Circuitry Mechanisms Regulating Adult Mammalian Neurogenesis. Cold Spring Harb Perspect Biol 8:a018937. doi:

10.1101/cshperspect.a018937

43. Sorrells SF, Paredes MF, Zhang Z, Kang G, Pastor-Alonso O, Biagiotti S, Page CE, Sandoval K, Knox A, Connolly A, Huang EJ, Garcia-Verdugo JM, Oldham MC, Yang Z, Alvarez-Buylla A (2021) Positive Controls in Adults and Children Support That Very Few, If Any, New Neurons Are Born in the Adult Human Hippocampus. J Neurosci 41:2554-2565. doi: 10.1523/JNEUROSCI.0676-20.2020

44. Suzuki T, Yamamoto M (2017) Stress-sensing mechanisms and the physiological roles of the Keap1Nrf2 system during cellular stress. J Biol Chem 292:16817-16824. doi: 10.1074/jbc.R117.800169

45. Tang Y, Le W (2016) Differential Roles of M1 and M2 Microglia in Neurodegenerative Diseases. Mol Neurobiol 53:1181-1194. doi: 10.1007/s12035-014-9070-5

46. Tobin MK, Musaraca K, Disouky A, Shetti A, Bheri A, Honer WG, Kim N, Dawe RJ, Bennett DA, Arfanakis K, Lazarov $O$ (2019) Human Hippocampal Neurogenesis Persists in Aged Adults and Alzheimer's Disease Patients. Cell Stem Cell 24:974-982e3. doi: 10.1016/j.stem.2019.05.003

47. Toda T, Parylak SL, Linker SB, Gage FH (2019) The role of adult hippocampal neurogenesis in brain health and disease. Mol Psychiatry 24:67-87. doi: 10.1038/s41380-018-0036-2

48. Valero J, Mastrella G, Neiva I, Sánchez S, Malva JO (2014) Long-term effects of an acute and systemic administration of LPS on adult neurogenesis and spatial memory. Front Neurosci 21:8-83. doi: $10.3389 /$ fnins.2014.00083

49. Vasan L, Park E, David LA, Fleming T, Schuurmans C (2021) Direct Neuronal Reprogramming: Bridging the Gap Between Basic Science and Clinical Application. Front Cell Dev Biol 9: 681087. doi: $0.3389 /$ fcell.2021.681087

50. Viola MF, Boeckxstaens G (2020) Intestinal resident macrophages: Multitaskers of the gut. Neurogastroenterol Motil 32:e13843. Doi: 10.1111/nmo.13843

51. Wang C, Cai X, Hu W, Li Z, Kong F, Chen X, Wang D (2019) Investigation of the neuroprotective effects of crocin via antioxidant activities in HT22 cells and in mice with Alzheimer's disease. Int $\mathrm{J}$ Mol Med 43:956-966. doi: 10.3892/ijmm.2018.4032

52. Xu Y, Yu L, Liu Y, Tang X, Wang X (2021) Lipopolysaccharide-Induced Microglial Neuroinflammation: Attenuation by FK866. Neurochem Res 46:1291-1304. doi: 10.1007/s11064-021-03267-4

53. Yang X, Yao C, Tian T, Li X, Yan H, Wu J, Li H, Pei L, Liu D, Tian Q, Zhu LQ, Lu Y (2018) A novel mechanism of memory loss in Alzheimer's disease mice via the degeneration of entorhinal-CA1 synapses. Mol Psychiatry 23:199-210. doi: 10.1038/mp.2016.151

54. Yeh CY, Asrican B, Moss J, Quintanilla LJ, He T, Mao X, Cassé F, Gebara E, Bao H, Lu W, Toni N, Song J (2018) Mossy Cells Control Adult Neural Stem Cell Quiescence and Maintenance through a Dynamic Balance between Direct and Indirect Pathways. Neuron 99:493-510e4. doi: 10.1016/j.neuron.2018.07.010

55. Yoo AS, Sun AX, Li L, Shcheglovitov A, Portmann T, Li Y, Lee-Messer C, Dolmetsch RE, Tsien RW, Crabtree GR (2011) MicroRNA-mediated conversion of human fibroblasts to neurons. Nature 
56. Yüzer A, Ayaz F, Ince M (2019) Immunomodulatory activities of zinc(II)phthalocyanine on the mammalian macrophages through p38 pathway: Potential ex vivo immunomodulatory PDT reagents. Bioorg Chem 92:103249. doi: 10.1016/j.bioorg.2019.103249

57. Zallo F, Gardenal E, Verkhratsky A, Rodríguez JJ (2018) Loss of calretinin and parvalbumin positive interneurones in the hippocampal CA1 of aged Alzheimer's disease mice. Neurosci Lett 681:19-25. doi: 10.1016/j.neulet.2018.05.027

58. Zong K, Liu X, Sun Z, Piao L, Xuan Y, Jin Y, Cui CA (2020) Macelignan inhibits the inflammatory response of microglia and regulates neuronal survival. J Neuroimmunol 339:577123. doi:

10.1016/j.jneuroim.2019.577123

59. Zonis S, Ljubimov VA, Mahgerefteh M, Pechnick RN, Wawrowsky K, Chesnokova V (2013) p21Cip restrains hippocampal neurogenesis and protects neuronal progenitors from apoptosis during acute systemic inflammation. Hippocampus 23:1383-1394. doi: 10.1002/hipo.22192

\section{Figures}

G2: Differentiation group, HT22 cells that were cultured with neuronal differentiation media for 24 hr.
G3: Unstimulated macrophage media induction during neuronal differentiation, HT22 cells were cultured
with a half mixture of unstimulated RAW 264.7 cell CM and neuronal differentiation media.
G5: Unstimulated macrophage CM induction after neuronal differentiation, upon 24 hr induction of neuronal
differentiation media, HT22 cells were treated with a half mixture of unstimulated RAW 264.7 CM and HG-
DMEM.

Figure 1 
Experimental cell culture design based on days and different culture media is presented. D (Day); G (group), HG-DMEM (High Glucose Dulbecco's modified Eagle's media); LPS (Lipopolysaccharide).

A)

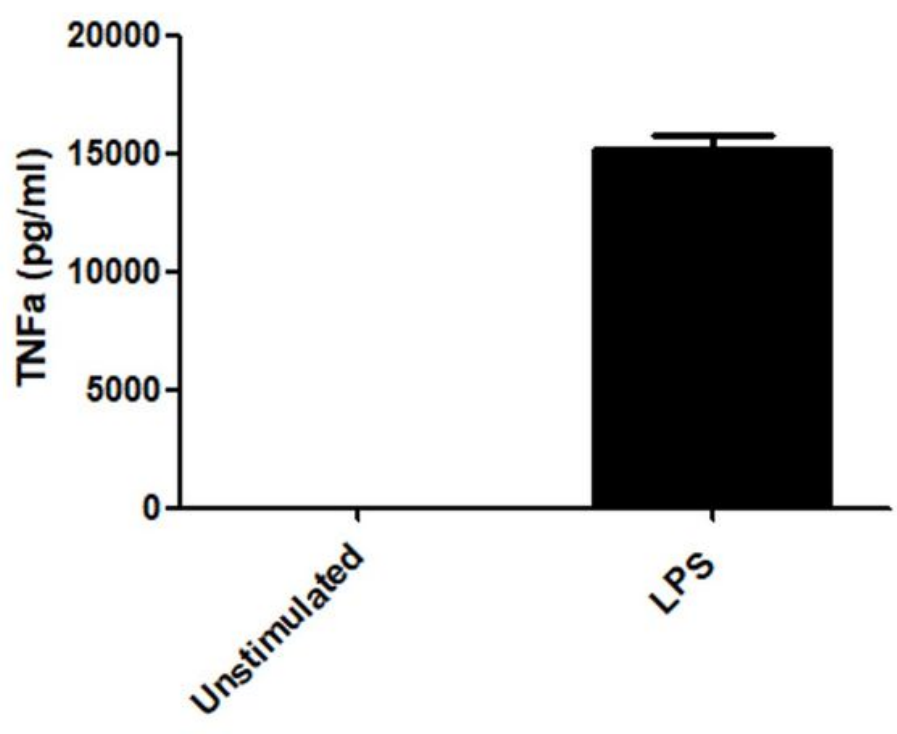

C)

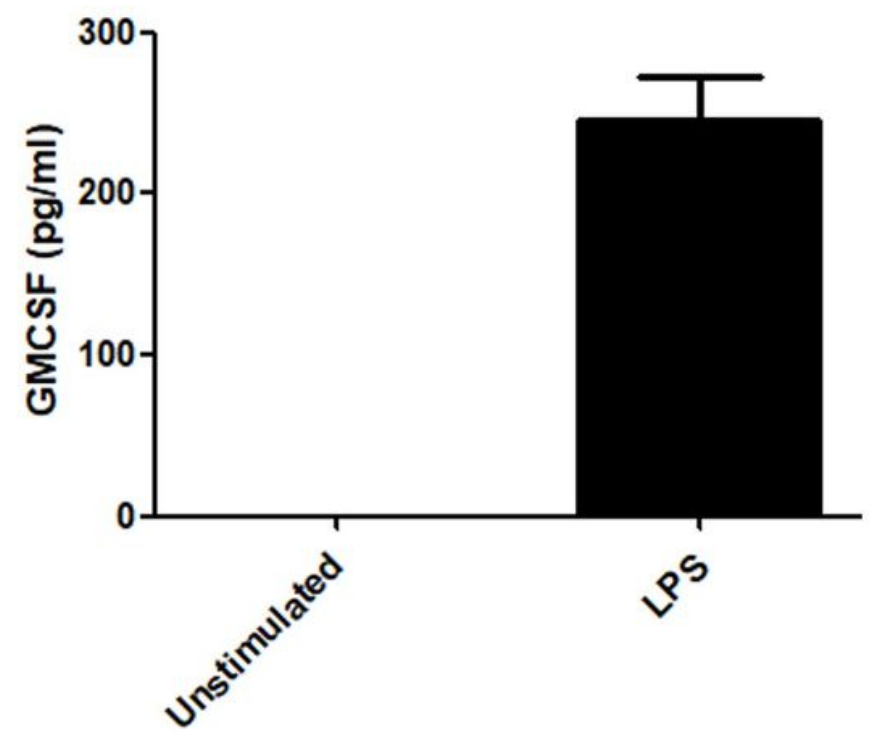

B)

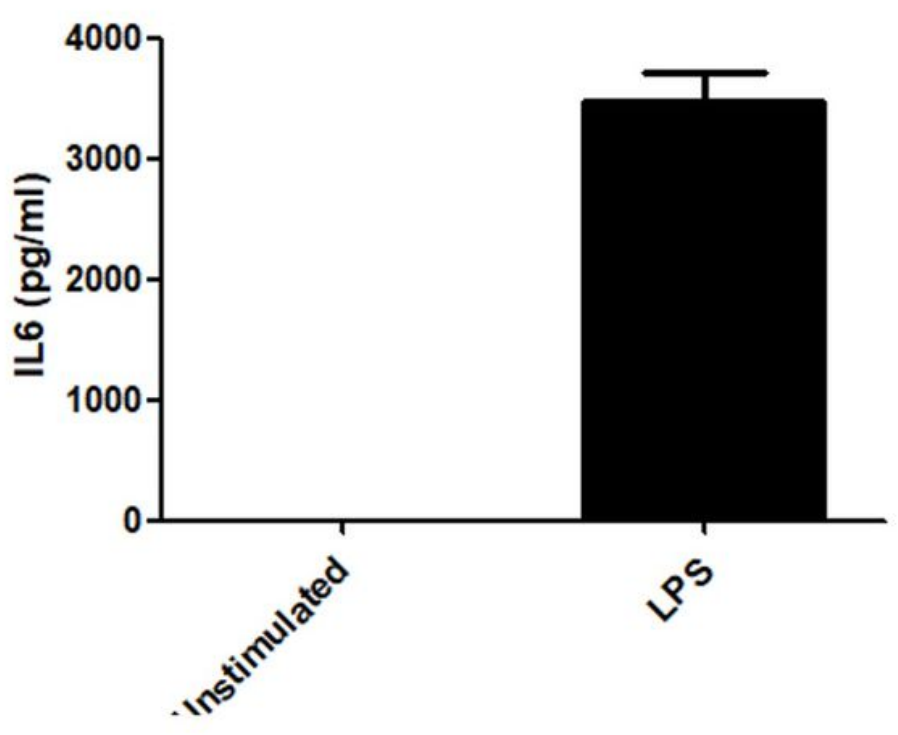

D)

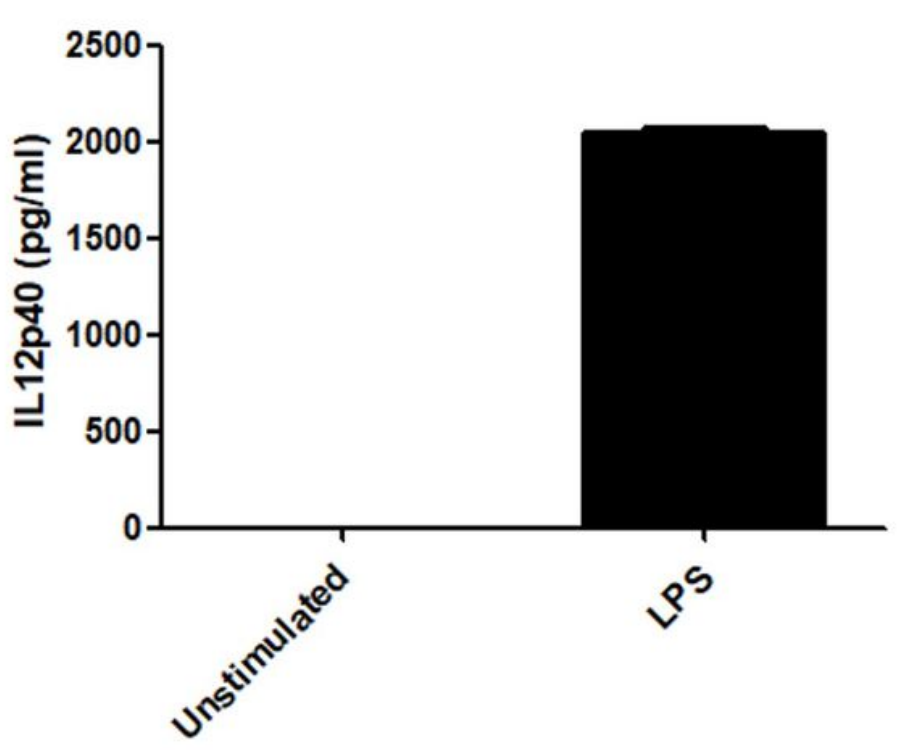

Figure 2

ELISA measurements of TNF-a (Tumor Necrosis Factor a), IL6 (Interleukin 6), GMCSF (GranulocyteMacrophage Colony-Stimulating Factor) and L12p40 (Interleukin-12 subunit p40), detected in picograms 
per milliliter $(\mathrm{pg} / \mathrm{ml})$ unit in the supernatant content of unstimulated and LPS-stimulated CM of RAW264.7 cells are shown in bar graphs, respectively (A-D).
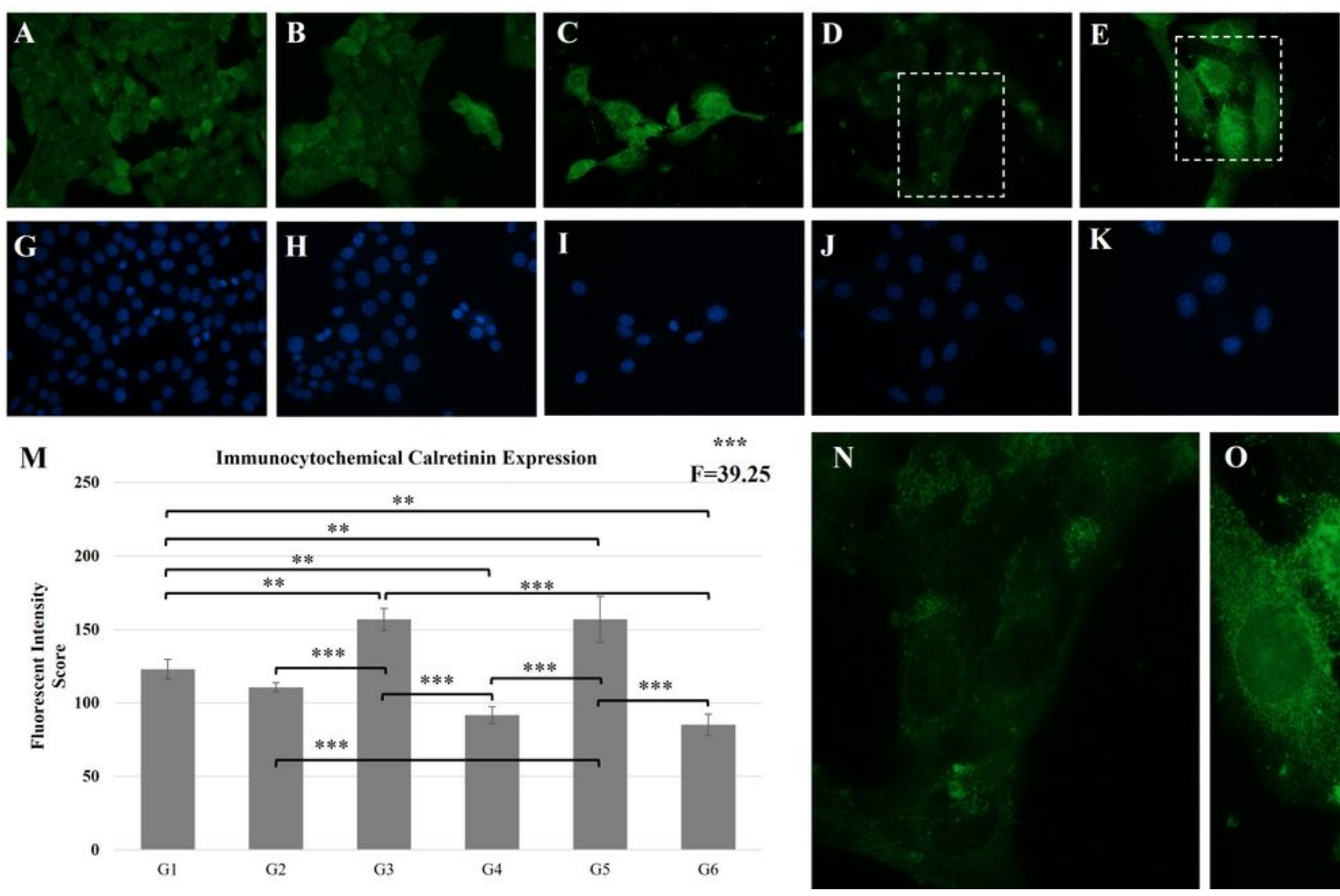

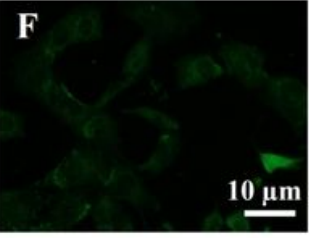

L

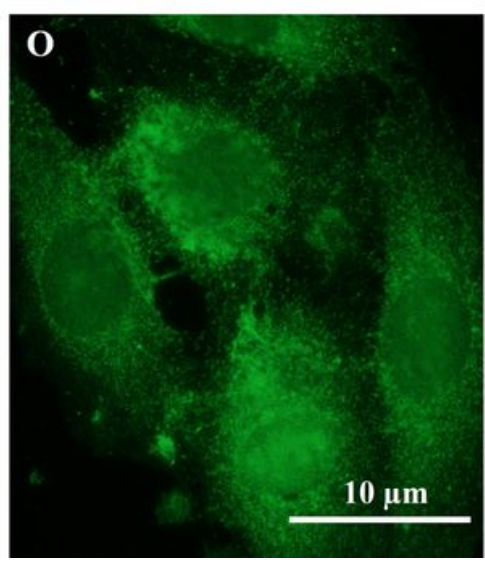

Figure 3

Intracellular CR expression in HT22 cells. Immunofluorescent CR (Alexa Fluor 488-green) and nucleus staining (DAPI-blue) in the study groups of HT22 cells (G1-G6, see Fig.1) are shown in A-F and G-L sections, respectively. Comparison of $\mathrm{CR}$ expression, based on the fluorescent intensity scores among the experimental groups (G1-G6) is shown in the bar graph (M). Error bars represent the standard deviation of intensity score of four different experiments (dishes) within the groups (M). Significance and $F$ values of ONE-Way ANOVA test are shown at the right upper corner of the graph $(\mathrm{M})$ which indicates whether differences exist among the mean intensity scores of different groups. Horizontal lines in between the bars indicate statistically significant $(p<0.05)$ pair-wise alternations, analyzed via Bonferroni post-hoc test. Pair-wise relations with significance level of $p>0.05$ were not shown. Magnification of the boxed areas in $D$ and $E$ are exhibited in separate visuals in $N$ and 0 , respectively. ${ }^{* \star p}<0.01,{ }^{\star \star *} p<0.001$. 


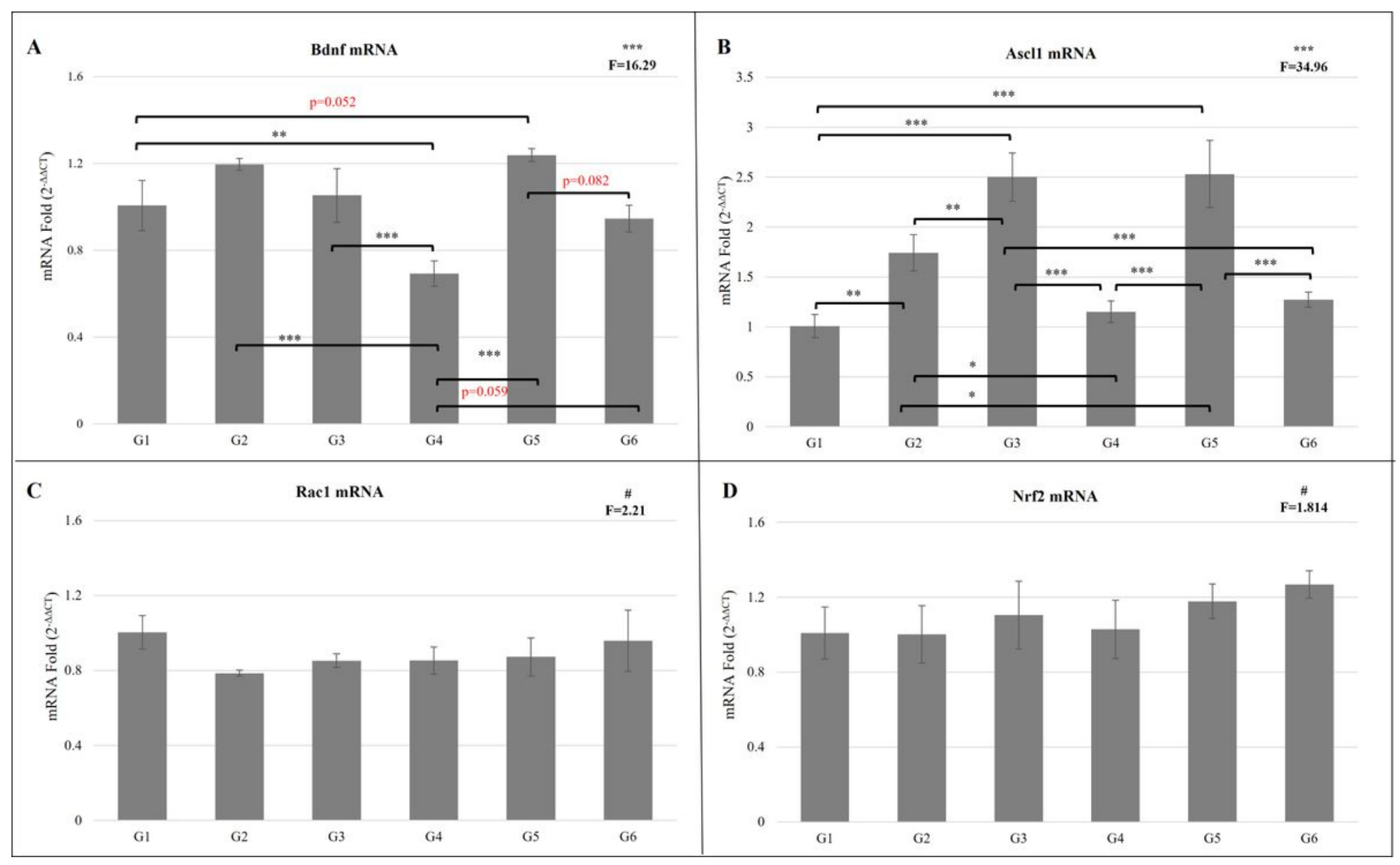

Figure 4

Q-RT-PCR analysis of Bdnf (A), Ascl1 (B), Rac1 (C) and Nrf2 (D) mRNA levels across the groups are shown. ${ }^{*} p>0.05,{ }^{\star} p<0.05,{ }^{* \star} p<0.01,{ }^{* \star *} p<0.001$. G1-G6 represents the groups given in the experimental cell culture design of the study (Fig.1). G1, control group; G2, differentiation group; G3, unstimulated macrophage CM induction during neuronal differentiation; G4, LPS induced macrophage CM induction during neuronal differentiation; $\mathbf{G 5}$, unstimulated macrophage $\mathrm{CM}$ induction after neuronal differentiation; G6, LPS induced macrophage CM induction after neuronal differentiation

\section{Supplementary Files}

This is a list of supplementary files associated with this preprint. Click to download.

- GraphicalAbstract.tif

- Supp.Fig..jpg 